\title{
Metabolic syndrome, C-reactive protein and microalbuminuria in a rural Chinese population: a cross-sectional study
}

\author{
Liping Jiang ${ }^{1}$, Wen Huang ${ }^{1 *}$, Yuanbo Liang ${ }^{2}$, Fenghua Wang ${ }^{2}$, Xinrong Duan², Xiaohui Yang ${ }^{2}$, Jiangping Wen ${ }^{3}$ \\ and Ningli Wang ${ }^{2 *}$
}

\begin{abstract}
Background: Microalbuminuria is an early marker of chronic kidney disease (CKD). Previous studies have shown that either metabolic syndrome (MetS) or chronic inflammation is related to renal impairment. The aim of this study was to investigate the association between MetS, C-reactive protein (CRP) and microalbuminuria in a rural Chinese population.

Methods: This was a cross-sectional study using data from the Handan Eye Study. MetS was defined according to the Chinese Diabetes Society (CDS) criteria. CRP levels $\geq 3 \mathrm{mg} / \mathrm{L}$ were classified as high CRP. Microalbuminuria was defined as a urinary albumin/creatinine ratio (ACR) of 30-300 mg/g.

Results: We included 4191 subjects aged $\geq 30$ years in this analysis. The prevalence of MetS and microalbuminuria in the group was $25.7 \%$ and $15.6 \%$, respectively. The odds ratio (OR) of microalbuminuria in subjects with MetS was 1.25 (95\% confidence interval (Cl): 1.03 - 1.51) compared with those without microalbuminuria. In multivariate logistic regression analysis, high blood pressure (OR 1.36, 95\% Cl: $1.10-1.67)$ and high fasting blood glucose (OR 1.44, 95\% Cl: $1.17-1.76$ ) were independently associated with microalbuminuria. Subjects with high CRP and MetS had a 1.46-fold greater risk of having microalbuminuria compared with those with low CRP without MetS (95\% Cl: $1.06-2.01)$.
\end{abstract}

Conclusions: In this rural Chinese population aged $\geq 30$ years, MetS and microalbuminuria were independently related and the combination of high CRP and MetS was associated with an increased risk of microalbuminuria.

Keywords: Microalbuminuria, Metabolic syndrome, Inflammation, Epidemiology

\section{Background}

Chronic kidney disease (CKD) has become a worldwide public health problem and microalbuminuria is an early marker of CKD. Many studies have shown that microalbuminuria is a strong and independent predictor of progressive kidney disease, cardiovascular events and all-cause mortality in diabetic, non-diabetic, hypertensive persons, and even in the general population [1-5]. Therefore, early prevention and treatment of microalbuminuria, by inhibiting the renin-angiotensin system, is generally

\footnotetext{
*Correspondence: huangw@trhos.com; wningli@vip.163.com

'Department of Nephrology, Beijing TongRen Hospital, Capital Medical University, Beijing, China

${ }^{2}$ Beijing TongRen Eye Center, Beijing TongRen Hospital, Capital Medical University, Beijing, China

Full list of author information is available at the end of the article
}

recommended in high risk patients, such as those with diabetes or hypertension [6]. Furthermore, identification and treatment of the risk factors for microalbuminuria may be an effective approach to prevent adverse outcomes.

Metabolic syndrome (MetS) is a common disorder characterized by abdominal obesity, hypertriglyceridemia, low high density lipoprotein (HDL) cholesterol level, high blood pressure (BP), and high fasting blood glucose level. A previous national cross-sectional study reported that the prevalence of MetS was $15.1 \%$ in Chinese adults aged 35-74 years [7]. Further, previous studies have shown that MetS is associated with microalbuminuria [8-10].

C-reactive protein (CRP) has been recognized as a sensitive marker of inflammation. Some cross-sectional studies have found a significant correlation between CRP and 
microalbuminuria in the general population $[11,12]$; however, other studies found conflicting results [13,14]. Studies examining the relationship between CRP and microalbuminuria in Chinese populations are rare. Recently, we reported that a high prevalence of microalbuminuria (15.3\%) was observed in a rural Chinese population aged $\geq 30$ years [15]. The aim of the current study was to investigate the association between MetS, CRP and the risk of microalbuminuria in the same rural Chinese population.

\section{Methods}

\section{Study population}

The Handan Eye Study (HES) was a population-based, cross-sectional study conducted from October 2006 to October 2007, which was designed to survey eye diseases and other health-related problems. Detailed information on this study has been published elsewhere [16]. In brief, residents of Yongnian County, Handan city, Hebei province, China, aged $\geq 30$ years were randomly selected using a stratified, clustered and multi-staged sampling technique. Yongnian County is located approximately $500 \mathrm{~km}$ south of Beijing and covers $980 \mathrm{~km}^{2}$. The total population was 830000 in 2005; $90 \%$ of the population were farmers; and 98\% were Han. Thirteen of the 458 villages in Yongnian County were randomly selected to achieve a target sample size of 8653, with 7557 participants considered eligible; 6830 underwent a comprehensive eye examination. Data from 4191 participants aged $\geq 30$ years were complete and included in this study. The HES protocol was approved by the Beijing TongRen Hospital Ethical Committee and conformed to the Declaration of Helsinki. All subjects gave written informed consent.

\section{Data collection}

Sociodemographic characteristics (age, sex and education), medical history (hypertension, diabetes and cardiovascular disease or stroke), and lifestyle behavior (smoking status and alcohol intake) were obtained using questionnaires. Height, weight and waist circumference were measured according to standard protocols. Body mass index (BMI) was calculated from weight and height measurements as weight $(\mathrm{kg})$ divided by the square of the height $\left(\mathrm{m}^{2}\right)$. BP was measured twice in a sitting position after at least 5 minutes' rest. An average of the two readings was then calculated. Blood specimens were collected after an overnight fast of at least 8 hours. Serum creatinine $(\mathrm{SCr})$ was measured using a kinetic Jaffe reaction. Glucose, triglycerides and HDL cholesterol were measured by enzymatic methods using an automatic clinical chemistry analyzer (Olympus AU2700, Tokyo, Japan). High-sensitivity CRP was measured using immunonephelometry with the IMMAGE Immunochemistry System (Beckman Coulter, Inc., Fullerton, CA, USA). Fresh midstream urine was collected in the morning and women who were actively menstruating were excluded from the urine test. Urine albumin concentration was measured by immunoturbidimetry (Audit Diagnostics, Cork, Ireland). Urine creatinine was measured by the same method used for SCr.

\section{Definitions}

MetS was confirmed according to the Chinese Diabetes Society (CDS) criteria when a subject had three or more of the following five components [17]: 1) obesity: BMI $\geq$ $25 \mathrm{~kg} / \mathrm{m}^{2}$; 2) fasting blood glucose $\geq 6.1 \mathrm{mmol} / \mathrm{L}$ or receiving antidiabetic medication; 3 ) triglycerides $\geq 1.7 \mathrm{mmol} / \mathrm{L}$; 4) $\mathrm{HDL}$ cholesterol $<1.04 \mathrm{mmol} / \mathrm{L}$; and 5) $\mathrm{BP} \geq 130 /$ $85 \mathrm{mmHg}$ or receiving antihypertensive medication. We also analyzed data on central obesity, defined as waist circumference $>90 \mathrm{~cm}$ in men or $>80 \mathrm{~cm}$ in women according to the American Heart Association/National Heart, Lung, and Blood Institute Scientific Statement (AHA/NHLBI) [18]. A CRP cutoff of $3 \mathrm{mg} / \mathrm{L}$ was used to differentiate high and low CRP groups according to the American Heart Association/Centers for Disease Control (AHA/CDC) consensus [19]. Subjects with clinical inflammation (CRP levels $>10 \mathrm{mg} / \mathrm{L}$ ) were excluded from this study. Urinary albumin/creatinine ratios (ACR) of 30$300 \mathrm{mg} / \mathrm{g}$ and $<30 \mathrm{mg} / \mathrm{g}$ were defined as microalbuminuria and normoalbuminuria, respectively. Participants with macroalbuminuria (ACR $>300 \mathrm{mg} / \mathrm{g}$ ) were excluded. We repeated the analysis using gender-specific definitions of microalbuminuria (17-250 mg/g for men and $25-355 \mathrm{mg} / \mathrm{g}$ for women) based on the National Kidney Foundation guidelines [20].

Hypertension was defined as systolic $\mathrm{BP} \geq 140 \mathrm{~mm} \mathrm{Hg}$ or diastolic $\mathrm{BP} \geq 90 \mathrm{~mm} \mathrm{Hg}$, or receiving antihypertensive medication. Diabetes was defined as fasting blood glucose $\geq 7.0 \mathrm{mmol} / \mathrm{L}$ or a self-reported history of diabetes.

\section{Statistical analysis}

All analyses were performed using SPSS software, version 11.5 (SPSS, Inc., Chicago, IL, USA). Data are presented as the mean \pm SD for continuous variables and as proportions for categorical variables. Appropriate variables were compared using the unpaired $t$ test for continuous data and $\chi^{2}$ test for categorical data. Because the CRP distribution was skewed, variables are expressed as medians and interquartile ranges and were compared using the Mann-Whitney test. We calculated the frequency of the individual MetS components, MetS and high CRP. The association between MetS, CRP and microalbuminuria was analysed using univariate and multivariate logistic regression analysis. The odds ratio (OR) of microalbuminuria was calculated according to the CRP levels and MetS status and a 95\% confidence interval (CI) was obtained using a multiple logistic 
regression model. We also used multivariate linear regression analysis to estimate the association between CRP levels (independent variable) and urinary ACR (dependent variable). All reported $p$ values are based on two-sided tests and $p<0.05$ was considered significant.

\section{Results}

\section{Baseline characteristics}

A total of 4191 participants were studied; $55.4 \%(n=2320)$ were female and the mean age was $51.7 \pm 11.1$ years. The prevalence of microalbuminuria in this rural Chinese population aged $\geq 30$ years was $15.6 \%$. Table 1 shows the baseline characteristics according to the microalbuminuria status. Compared with the normoalbuminuria group, the microalbuminuria group had a greater age; higher waist circumference; history of cardiovascular disease; and higher systolic BP; diastolic BP; fasting blood glucose; triglycerides; and CRP levels. Hypertension, diabetes and female gender were prevalent among subjects with microalbuminuria.

A total of 1076 (25.7\%) subjects had MetS; 41.4\% were obese; $65.0 \%$ had high BP; $19.5 \%$ had high fasting glucose; 28.3\% had high triglycerides; and $19.0 \%$ had low HDL cholesterol. High CRP ( $\geq 3 \mathrm{mg} / \mathrm{L}$ ) was observed in 660 (15.7\%) subjects. The prevalence of MetS and high CRP was higher in subjects with microalbuminuria than in those with normoalbuminuria ( $31.1 \%$ vs. $24.7 \%, p=0.001$ and $19.1 \%$ vs. $15.1 \%, p=0.01)$.

\section{Association between MetS, CRP and microalbuminuria}

Table 2 shows the ORs for microalbuminuria by individual MetS component, MetS and high CRP. In the unadjusted regression analysis, high BP (OR 1.76, 95\% CI: 1.45 - 2.12) and high fasting blood glucose (OR 1.65, 95\% CI: 1.36 -

Table 1 Basic patient characteristics according to microalbuminuria status

\begin{tabular}{|c|c|c|c|c|}
\hline & $\begin{array}{l}\text { Total } \\
\mathrm{n}=4191\end{array}$ & $\begin{array}{l}\text { Normoalbuminuria } \\
\mathrm{n}=3538\end{array}$ & $\begin{array}{l}\text { Microalbuminuria * } \\
n=653\end{array}$ & $p$ value \\
\hline Age (y) & $51.7 \pm 11.1$ & $51.1 \pm 11.1$ & $54.7 \pm 10.7$ & $<0.001$ \\
\hline Female, No. (\%) & $2320(55.4)$ & $1919(54.2)$ & $401(61.4)$ & 0.001 \\
\hline Current smoker, No. (\%) & $1078(26.0)$ & $929(26.5)$ & $149(23.2)$ & 0.09 \\
\hline Current drinker, No. (\%) & $774(18.7)$ & $659(18.8)$ & 115(18.0) & 0.63 \\
\hline High school education, No. (\%) & $132(3.2)$ & $110(3.1)$ & $22(3.4)$ & 0.71 \\
\hline Cardiovascular disease, No. (\%) & 207(4.9) & $162(4.6)$ & $45(7.0)$ & 0.01 \\
\hline Stroke, No. (\%) & $101(2.4)$ & $89(2.5)$ & $12(1.9)$ & 0.32 \\
\hline $\mathrm{BMI}\left(\mathrm{kg} / \mathrm{m}^{2}\right)$ & $24.7 \pm 3.7$ & $24.7 \pm 3.7$ & $24.7 \pm 3.8$ & 0.96 \\
\hline Waist circumference $(\mathrm{cm})$ & $87.9 \pm 9.6$ & $87.7 \pm 9.6$ & $88.8 \pm 9.5$ & 0.009 \\
\hline Systolic BP (mm Hg) & $139.4 \pm 22.1$ & $138.1 \pm 21.5$ & $146.4 \pm 24.4$ & $<0.001$ \\
\hline Diastolic BP(mm Hg) & $77.7 \pm 12.2$ & $77.3 \pm 12.0$ & $80.1 \pm 13.2$ & $<0.001$ \\
\hline Fasting glucose (mmol/L) & $5.8 \pm 1.3$ & $5.7 \pm 1.2$ & $6.1 \pm 1.9$ & $<0.001$ \\
\hline Triglycerides (mmol/L) & $1.5 \pm 1.0$ & $1.5 \pm 0.9$ & $1.6 \pm 1.3$ & $<0.001$ \\
\hline HDL cholesterol (mmol/L) & $1.3 \pm 0.3$ & $1.3 \pm 0.2$ & $1.3 \pm 0.3$ & 0.33 \\
\hline $\mathrm{SCr}$ (umol/L) & $71.4 \pm 10.8$ & $71.5 \pm 10.7$ & $70.8 \pm 11.3$ & 0.11 \\
\hline Diabetes, No. (\%) & $259(6.2)$ & $195(5.5)$ & $64(9.8)$ & $<0.001$ \\
\hline Hypertension, No. (\%) & $2076(49.5)$ & $1672(47.3)$ & $404(61.9)$ & $<0.001$ \\
\hline $\mathrm{CRP}(\mathrm{mg} / \mathrm{L})$ & $0.8(0.4-2.1)$ & $0.8(0.4-2.0)$ & $1.0(0.4-2.3)$ & 0.01 \\
\hline High CRP ${ }^{+}$, No. (\%) & $660(15.7)$ & $535(15.1)$ & $125(19.1)$ & 0.01 \\
\hline Obesity, No. (\%) & $1737(41.4)$ & $1467(41.5)$ & $270(41.3)$ & 0.96 \\
\hline High BP, No. (\%) & $2723(65.0)$ & 2233(63.1) & $490(75.0)$ & $<0.001$ \\
\hline High fasting glucose, No. (\%) & $818(19.5)$ & $643(18.2)$ & $175(26.8)$ & $<0.001$ \\
\hline High triglycerides, No. (\%) & $1185(28.3)$ & $982(27.8)$ & 203(31.1) & 0.08 \\
\hline Low HDL cholesterol, No. (\%) & $797(19.0)$ & $677(19.1)$ & $120(18.4)$ & 0.65 \\
\hline MetS ${ }^{\ddagger}$, No. (\%) & $1076(25.7)$ & $873(24.7)$ & $203(31.1)$ & 0.001 \\
\hline
\end{tabular}

$B M I$ body mass index, BP blood pressure, $C R P$ C-reactive protein, $H D L$ high density lipoprotein, MetS metabolic syndrome, $S C r$ serum creatinine.

* Microalbuminuria was defined as a urinary albumin/creatinine ratio (ACR) of $30-300 \mathrm{mg} / \mathrm{g}$.

${ }^{+}$High CRP was defined as a CRP $\geq 3 \mathrm{mg} / \mathrm{dl}$.

${ }^{\ddagger}$ MetS was defined by the Chinese Diabetes Society (CDS). 
Table 2 Odds ratios for microalbuminuria by individual MetS component, MetS and CRP

\begin{tabular}{|c|c|c|c|c|c|c|}
\hline & \multicolumn{2}{|c|}{ Unadjusted } & \multicolumn{2}{|c|}{ Adjusted model1* } & \multicolumn{2}{|c|}{ Adjusted model $2^{\dagger}$} \\
\hline & OR (95\% Cl) & $p$ value & OR $(95 \% \mathrm{Cl})$ & $\overline{p \text { value }}$ & OR $(95 \% \mathrm{Cl})$ & $\overline{p \text { value }}$ \\
\hline Obesity & $1.00(0.84-1.18)$ & 0.96 & $0.98(0.82-1.17)$ & 0.81 & $0.92(0.76-1.10)$ & 0.34 \\
\hline High BP & $1.76(1.45-2.12)$ & $<0.001$ & $1.38(1.13-1.70)$ & 0.002 & $1.36(1.10-1.67)$ & 0.004 \\
\hline High fasting glucose & $1.65(1.36-1.99)$ & $<0.001$ & $1.47(1.20-1.80)$ & $<0.001$ & $1.44(1.17-1.76)$ & $<0.001$ \\
\hline High triglycerides & $1.17(0.98-1.41)$ & 0.08 & $1.10(0.91-1.32)$ & 0.35 & $1.03(0.85-1.26)$ & 0.76 \\
\hline Low HDL cholesterol & $1.95(0.77-1.18)$ & 0.65 & $1.03(0.83-1.29)$ & 0.77 & $1.04(0.82-1.30)$ & 0.76 \\
\hline High CRP & $1.33(1.07-1.65)$ & 0.01 & $1.16(0.93-1.45)$ & 0.19 & $1.12(0.89-1.40)$ & 0.34 \\
\hline MetS $^{\ddagger}$ & $1.38(1.15-1.65)$ & 0.001 & $1.25(1.03-1.51)$ & 0.02 & - & - \\
\hline
\end{tabular}

$B P$ blood pressure, $C R P$ C-reactive protein, $C l$ confidence interval, $H D L$ high density lipoprotein, MetS metabolic syndrome, $O R$ odds ratio.

* Adjusted for age, sex, smoking status, alcohol use, education level, history of cardiovascular disease or stroke and CRP (except for analyses on the High CRP).

${ }^{\dagger}$ Additionally adjusted for the other components of MetS.

* Compared with those without MetS (<3 components).

1.99) were associated with microalbuminuria. After adjusting for age, sex, smoking status, alcohol use, education level, history of cardiovascular disease or stroke and CRP, the associations were still significant. Adjusting for the components of MetS had little effect on the ORs. In the multivariate logistic regression model, MetS was independently associated with microalbuminuria (OR 1.25, 95\% CI: $1.03-1.51, p=0.02$ ); however, the association between high CRP and microalbuminuria disappeared after adjustments were made for age, sex, and other possible risk factors for CKD $(p=0.34)$. A similar result was observed in the multivariate linear regression analysis; after adjusting for age, sex and the components of MetS, there was no relationship between CRP and urinary ACR $(p=0.78)$.

\section{Interrelationship between MetS, CRP and microalbuminuria}

Table 3 shows the ORs for microalbuminuria by CRP levels and MetS status. The prevalence of microalbuminuria in each group was as follows: low CRP without MetS, 14.2\%; high CRP without MetS, 16.3\%; low CRP with MetS, 17.6\%; and high CRP with MetS, 22.8\%. There was no significant difference between the low CRP without MetS and high CRP without MetS groups (14.2\% vs. $16.3 \%, p=0.26)$ as well as between the low CRP with MetS and high CRP with MetS groups $(17.6 \%$ vs. $22.8 \%, p=0.06)$. Compared with the low CRP without MetS group, the multivariate adjusted OR for microalbuminuria was 1.23 (95\% CI: $0.99-1.52)$ and 1.05 (95\% CI: $0.78-1.42)$ in the high CRP without MetS and low CRP with MetS groups, respectively. Subjects with high CRP and MetS had a 1.46 -fold increase in OR for microalbuminuria $(95 \% \mathrm{CI}$ : $1.06-2.01, p=0.02$ ).

\section{Sensitivity analysis}

First, we included 4180 subjects with complete information in the analysis of central obesity. The prevalence of MetS and microalbuminuria was 30.1\% $(\mathrm{n}=1259)$ and $15.5 \%(n=649)$, respectively and there was a strong and independent relationship between MetS and microalbuminuria after further adjustments (OR 1.40, 95\% CI: $1.17-1.68, p<0.001$ ).

Second, the results were similar when gender-specific microalbuminuria cutoffs were applied; a total of 1076 (23.2\%) subjects had microalbuminuria. In the multivariateadjusted model, MetS was independently associated with microalbuminuria (OR 1.23, 95\% CI: $1.05-1.45, p=0.01$ ), and we found no relationship between CRP and microalbuminuria $(p=0.14)$. Subjects with high CRP and MetS had a 1.37-fold greater risk of having microalbuminuria compared with those with low CRP without MetS (95\% CI: $1.03-1.82, p=0.03$ ).

Third, after excluding subjects with hypertension, the prevalence of MetS and microalbuminuria decreased

Table 3 Odds ratios for microalbuminuria by CRP levels and MetS status

\begin{tabular}{|c|c|c|c|c|c|c|}
\hline & \multirow[t]{2}{*}{$\mathrm{N}$} & \multirow{2}{*}{$\begin{array}{l}\text { Microalbuminuria } \\
\text { N. (\%) }\end{array}$} & \multicolumn{2}{|c|}{ Unadjusted } & \multicolumn{2}{|c|}{ Multivariate-adjusted $^{*}$} \\
\hline & & & OR $(95 \% \mathrm{Cl})$ & $p$ value & OR $(95 \% \mathrm{Cl})$ & $p$ value \\
\hline Low CRP without MetS & 2723 & $386(14.2)$ & reference & & reference & \\
\hline High CRP without MetS & 392 & $64(16.3)$ & $1.29(1.05-1.59)$ & 0.02 & 1.23(0.99-1.52) & 0.07 \\
\hline Low CRP with MetS & 808 & $142(17.6)$ & 1.09(0.91-1.26) & 0.26 & 1.05(0.78-1.42) & 0.74 \\
\hline High CRP with MetS & 268 & $61(22.8)$ & $1.78(1.32-2.42)$ & $<0.001$ & $1.46(1.06-2.01)$ & 0.02 \\
\hline
\end{tabular}

CRP C-reactive protein, $\mathrm{Cl}$ confidence interval, MetS metabolic syndrome, OR odds ratio.

${ }^{*}$ Adjusted for age, sex, smoking status, alcohol use, education level, history of cardiovascular disease or stroke. 
substantially from $25.7 \%$ to $12.8 \%$ and from $15.6 \%$ to $11.8 \%$, respectively and MetS was not significantly associated with microalbuminuria (OR 1.01, 95\% CI: $0.67-1.53, p=0.98)$.

\section{Discussion}

In this rural Chinese population aged $\geq 30$ years, we found that MetS, as defined by the CDS, was independently associated with microalbuminuria after adjusting for age, sex and other potential confounding factors. High BP and high fasting blood glucose were the two main risk factors. In addition, we found that CRP was not related to microalbuminuria in the multivariate analysis; however, the combination of high CRP and MetS increased the risk of microalbuminuria.

We used ACR to assess microalbuminuria in our study. The prevalence of microalbuminuria in this rural population was $15.6 \%$, higher than other population-based studies in the US (6.4\% in 5659 adults aged $20-80$ years in the National Health and Nutrition Examination Survey (NHANES III)) [8], Japan (13.7\% in 2321 participants aged 40-87 years) [9] and China (5.3 - 11.5\%) [10,21-24]. Notably, the reports from Chinese urban regions showed that the prevalence of microalbuminuria was $5.3 \%$ in Beijing (2310 adults aged $\geq 40$ years) [21]; 5.8\% in Guangzhou (6311 adults aged $\geq 20$ years) [22]; 6.7\% in Shanghai (3532 adults aged $\geq 20$ years) [23]; $8.8 \%$ in Hangzhou (2969 adults aged $18-87$ years) [24]; and $11.5 \%$ in Taiwan (2311 adults aged $\geq 40$ years) [10]. The discrepancy may be due to differences in the sample size and population studied, including factors such as age, race, region or other factors.

The prevalence of MetS in our study was $25.7 \%$, lower than two urban population studies in Beijing (34.1\%) [21] and Taiwan (39.1\%) [10], and higher than a recent study in Hangzhou (12.6\%) [24]. The difference between urban and rural populations may be partly due to different age groups, geographic regions or definitions of MetS. Our study identified a positive relationship between MetS and microalbuminuria in the rural Chinese adult population, which was compatible with previous studies. Nevertheless, the association between the specific MetS components and microalbuminuria in these populations was inconsistent. Studies in the US and Japan reported that high BP, high fasting blood glucose and obesity were associated with microalbuminuria [8,9]. The results from two Chinese studies demonstrated that all MetS components were associated with microalbuminuria $[10,24]$. In our study, high BP and high fasting blood glucose were independent risk factors for microalbuminuria, and no association between other MetS components and microalbuminuria was seen. A similar result was obtained in a Chinese urban population study [23]. High BP and high fasting blood glucose are two important components of MetS. As previously reported, our study revealed that mildly elevated BP ( $\geq 130 / 85 \mathrm{~mm} \mathrm{Hg}$ ) or fasting blood glucose levels $(\geq 6.1 \mathrm{mmol} / \mathrm{l}$ ) were independently associated with microalbuminuria. Based on these findings, we can easily identify subjects who are at risk of microalbuminuria by measuring BP and fasting blood glucose level. In addition, sensitivity analysis revealed that the observed correlation between MetS and microalbuminuria was strongly affected by the presence of hypertension, which may be attributed to the high prevalence of hypertension (49.5\%) in the present study, higher than previous reports from Beijing (47.1\%) [21] and southern China (19.2\%) [22]. The reason for this may be the different age groups, cardiovascular risk factors or lifestyle and dietary patterns which could potentially affect blood pressure. Hypertension may be an important threat for microalbuminuria in this rural population.

Although several epidemiological studies have confirmed the relationship between MetS and microalbuminuria, the underlying mechanisms are still not completely understood. Several possible reasons have been proposed, including insulin resistance, inflammation, renal endothelial dysfunction, altered renal hemodynamics, and others [25]. The results of our and other studies have shown that CRP, a widely used marker of chronic inflammation, was strongly related to MetS [26-29]. Data from population-based studies from the US [11], Japan [12] and Singapore [30] have shown that CRP was associated with microalbuminuria. These findings suggest that inflammation may play an important role in early kidney damage. However, even though $15.7 \%$ of subjects had high CRP levels ( $\geq 3 \mathrm{mg} / \mathrm{L}$ ) in our study, a relationship between high CRP and microalbuminuria was not seen. This is similar to previous reports showing that CRP was associated with decreased renal function but not albuminuria [13], or that microalbuminuria was related to a different marker of inflammation (e.g., fibrinogen) but not CRP in a logistic regression model [14]. We also examined the interrelationship between CRP and MetS and the results showed that only the combination of high CRP and MetS was independently associated with microalbuminuria (OR 1.46, 95\% CI: 1.05 - 2.01) after further adjustments. Our findings are supported by a recent study indicating that the odds for CKD (defined as an estimated glomerular filtration rate $\left(\right.$ eGFR) $<60 \mathrm{ml} / \mathrm{min} / 1.73 \mathrm{~m}^{2}$ ) increased only in the subgroup having both high CRP and MetS [31]. In another study, CRP was associated with CKD (defined as an eGFR $<60 \mathrm{ml} / \mathrm{min} / 1.73 \mathrm{~m}^{2}$ or albuminuria) independent of MetS, and subjects with high CRP and MetS had the highest odds for CKD of the four groups [32]. These results suggest a positive interrelationship between these MetS and CRP, and microalbuminuria. One consideration is that inflammation and 
MetS had synergistic or additive effects on renal damage.

This study had a number of limitations. First, a causal relationship cannot be established between MetS and microalbuminuria because of the cross-sectional study design. Second, the ACR measurement was based on a single spot urine sample, which could result in misclassification of the microalbuminuria status. However, this simple sampling procedure was commonly used in epidemiologic studies. Third, several studies have reported that statin drugs may decrease the CRP levels [33,34]. We did not control for the effect of statin drugs in this study, because the number of statin users was relatively small. Only $1.7 \%$ $(\mathrm{n}=71)$ of participants answered whether lipid-lowering drugs were being taken during the study period. Finally, selection bias might, in theory, have influenced our results.

\section{Conclusions}

In conclusion, our study showed that MetS was associated with microalbuminuria in a rural Chinese population aged $\geq 30$ years. Despite a lack of an independent association between CRP and microalbuminuria, high CRP combined with MetS increased the risk of microalbuminuria. Further prospective cohort studies are necessary to clarify these causal relationships.

\section{Abbreviations}

ACR: Albumin/creatinine ratio; BMI: Body mass index; BP: Blood pressure; CDS: Chinese Diabetes Society; Cl: Confidence interval; CKD: Chronic kidney disease; CRP: C-reactive protein; eGFR: Estimated glomerular filtration rate; HDL: High density lipoprotein; MetS: Metabolic syndrome; OR: Odds ratio; SCr: Serum creatinine.

\section{Competing interests}

The authors declare that they have no competing interests.

\section{Authors' contributions}

$N W, Y L, F W, X D, X Y, L J$ and $W H$ participated in the study design. YL, FW, XD, $X Y$ and $\amalg$ collected the data. JW performed the CRP assays. $L$ and WH contributed to the data analysis and manuscript preparation. All authors read and approved the final manuscript.

\section{Acknowledgments}

This study was supported by the National Basic Research Program of China (973rd Program), the Ministry of Science and Technology of China (Grant 2007 CB 512201), and the Handan Bureau of Science \& Technology. Additional support was provided by Beijing TongRen Hospital (Grant 2005).

\section{Author details}

'Department of Nephrology, Beijing TongRen Hospital, Capital Medical University, Beijing, China. ${ }^{2}$ Beijing TongRen Eye Center, Beijing TongRen Hospital, Capital Medical University, Beijing, China. ${ }^{3}$ Department of Laboratory Medicine, Beijing TongRen Hospital, Capital Medical University, Beijing, China.

Received: 21 October 2012 Accepted: 30 May 2013

Published: 2 June 2013

\section{References}

1. Gerstein HC, Mann JF, Yi Q, et al: Albuminuria and risk of cardiovascular events, death and heart failure in diabetic and nondiabetic individuals. JAMA 2001, 286:421-426.
2. Pinto-Sietsma SJ, Janssen WM, Hillege HL, et al: Urinary albumin excretion is associated with renal functional abnormalities in a nondiabetic population. J Am Soc Nephrol 2000, 11:1882-1888.

3. Pedrinelli R, Dell'Omo G, Di Bello V, et al: Microalbuminuria, an integrated marker of cardiovascular risk in essential hypertension. $J$ Hum Hypertens 2002, 16:79-89.

4. Hillege HL, Fidler V, Diercks GF, et al: Urinary albumin excretion predicts cardiovascular and noncardiovascular mortality in general population. Circulation 2002, 106:1777-1782.

5. Solbu MD, Kronborg J, Jenssen TG, et al: Albuminuria, metabolic syndrome and the risk of mortality and cardiovascular events. Atherosclerosis 2009, 204:503-508.

6. Palmer AJ, Roze S, Rodby RA, et al: Clinical and health economic implications of early treatment with irbesartan of patients with type 2 diabetes mellitus, hypertension and nephropathy. Dtsch Med Wochenschr 2006, 131:1721-1726.

7. Gu D, Reynolds K, Wu X, et al: Prevalence of the metabolic syndrome and overweight among adults in China. Lancet 2005, 365:1398-1405.

8. Palaniappan L, Carnethon M, Fortmann SP, et al: Association between microalbuminuria and the metabolic syndrome: NHANESIII. Am J Hypertens 2003, 16:952-958.

9. Hao Z, Konta T, Takasaki S, et al: The association between microalbuminuria and metabolic syndrome in the general population in Japan: the Takahata study. Inter Med 2007, 46:341-346.

10. Lin CC, Liu CS, Li TC, et al: Microalbuminuria and the metabolic syndrome and its components in the Chinese population. Eur J Clin Invest 2007, 37:783-790.

11. Kshirsagar AV, Bomback AS, Bang $H$, et al: Association of C-reactive protein and microalbuminuria (from the National Health and Nutrition Examination Surveys, 1999 to 2004). Am J Cardiol 2008, 101:401-406,

12. Nakamura M, Onoda T, Itai K, et al: Association between serum C-reactive protein levels and microalbuminuria: a population-based cross-sectional study in northern Iwate, Japan. Intern Med 2004, 43:919-925.

13. Fox ER, Benjamin EJ, Sarpong DF, et al: The relation of C-reactive protein to chronic kidney disease in African Americans:the Jackson Heart Study. BMC Nephrol 2010, 11:1.

14. Festa A, D'Agostino RD, Howard G, et al: Inflammation and microalbuminuria in nondiabetic and type 2diabetic subjects: The Insulin Resistance Atherosclerosis Study. Kidney Int 2000, 58:1703-1710.

15. Jiang L, Liang Y, Qiu B, et al: Prevalence of chronic kidney disease in a rural Chinese adult population: the Handan Eye Study. Nephron Clin Pract 2010, 114:295-302.

16. Liang YB, Friedman DS, Wong TY, et al: Prevalence and cause of low vision and blindness in a rural Chinese adult population: the handan eye study. Ophthalmology 2008, 115:1965-1972.

17. Expert Panel on Metabolic Syndrome of Chinese Diabetes Society: Recommendations on metabolic syndrome of Chinese Diabetes Society (Chinese). Chin J Diabetes 2004, 12:156-161.

18. Grundy SM, Cleeman Jl, Daniels SR, et al: Diagnosis and management of the metabolic syndrome: an American Heart Association/National Heart, Lung, and Blood Institute Scientific Statement. Circulation 2005, 112:2735-2752.

19. Pearson TA, Mensah GA, Alexander RW, et al: Markers of inflammation and cardiovascular disease: application to clinical and public health practice: a statement for healthcare professionals from the Centers for Disease Control and Prevention and the American Heart Association. Circulation 2003, 107:499-511.

20. Levey AS, Coresh J, Balk E, et al: National Kidney Foundation practice guidelines for chronic kidney disease: evaluation, classification, and stratification. Ann Intern Med 2003, 139:137-147.

21. Zhang L, Zuo L, Wang F, et al: Metabolic syndrome and chronic kidney disease in a Chinese population aged 40 years and older. Mayo Clin Proc 2007, 82:822-827.

22. Chen W, Chen W, Wang $H$, et al: Prevalence and risk factors associated with chronic kidney disease in an adult population from southern China. Nephrol Dial Transplant 2009, 24:1205-1212.

23. Li Q, Jia WP, Lu JQ, et al: Relationship between the prevalence of microalbuminuria and components of metabolic syndrome in Shanghai. Clin J Epidemiol 2004, 25:65-68.

24. Chen B, Yang D, Chen Y, et al: The prevalence of microalbuminuria and its relationships with the components of metabolic syndrome in the general population of China. Clin Chim Acta 2010, 411:705-709. 
25. Raimundo M, Lopes JA: Metabolic Syndrome, Chronic Kidney Disease, and Cardiovascular Disease: A Dynamic and Life-Threatening Triad. Cardiol Res Pract 2011, 2011:747-861.

26. Fröhlich M, Imhof A, Berg G, et al: Association between C-reactive protein and features of the metabolic syndrome: a population-based study. Diabetes Care 2000, 23:1835-1839.

27. Beddhu S, Kimmel PL, Ramkumar N, Cheung AK: Associations of metabolic syndrome with inflammation in CKD: results From the Third National Health and Nutrition Examination Survey (NHANES III). Am J Kidney Dis 2005, 46:577-586.

28. Ye X, Yu Z, Li H, et al: Distributions of C-reactive protein and its association with metabolic syndrome in middle aged and older Chinese people. J Am Coll Cardiol 2007, 49:1798-1805

29. Wen J, Liang Y, Wang F, et al: Association of C-reactive protein and metabolic syndrome in a rural Chinese population. Clin Biochem 2009 42:976-983.

30. Sabanayagam C, Lee J, Shankar A, et al: C-reactive protein and microalbuminuria in a multi- ethnic Asian population. Nephrol Dial Transplant 2010, 25:1167-1172.

31. Fakhrzadeh $H$, Ghaderpanahi M, Sharifi F, et al: Increased risk of chronic kidney disease in elderly with metabolic syndrome and high levels of Creactive protein: Kahrizak Elderly Study. Kidney Blood Press Res 2009, 32:457-463.

32. Lee JE, Choi SY, Huh W, et al: Metabolic syndrome, C-reactive protein, and chronic kidney disease in nondiabetic, nonhypertensive adults. Am J Hypertens 2007, 20:1189-1194.

33. Albert MA, Danielson E, Rifai N, Ridker PM, PRINCE Investigators: Effect of statin therapy on C-reactive protein levels: the Pravastatin Inflammation / CRP Evaluation (PRINCE): a randomized trial and cohort study. JAMA 2001, 286:64-70

34. Nissen SE, Tuzcu EM, Schoenhagen P, et al: Statin therapy, LDL cholesterol, C-reactive protein, and coronary artery disease. N Engl J Med 2005, 352:29-38.

\section{doi:10.1186/1471-2369-14-118}

Cite this article as: Jiang et al:: Metabolic syndrome, C-reactive protein and microalbuminuria in a rural Chinese population: a cross-sectional study. BMC Nephrology 2013 14:118.

\section{Submit your next manuscript to BioMed Central and take full advantage of:}

- Convenient online submission

- Thorough peer review

- No space constraints or color figure charges

- Immediate publication on acceptance

- Inclusion in PubMed, CAS, Scopus and Google Scholar

- Research which is freely available for redistribution 